\title{
Single-Shot Diffractive Imaging with a Table-Top Femtosecond Soft X-Ray Laser-Harmonics Source
}

\author{
A. Ravasio, ${ }^{1}$ D. Gauthier, ${ }^{1}$ F. R. N. C. Maia, ${ }^{2}$ M. Billon, ${ }^{1}$ J-P. Caumes, ${ }^{1}$ D. Garzella, ${ }^{1}$ M. Géléoc, ${ }^{1}$ O. Gobert, ${ }^{1}$ J-F. Hergott, ${ }^{1}$ \\ A-M. Pena, ${ }^{1}$ H. Perez, ${ }^{1}$ B. Carré, ${ }^{1}$ E. Bourhis, ${ }^{3}$ J. Gierak, ${ }^{3}$ A. Madouri, ${ }^{3}$ D. Mailly, ${ }^{3}$ B. Schiedt, ${ }^{3}$ M. Fajardo, ${ }^{4}$ J. Gautier, ${ }^{5}$ \\ P. Zeitoun, ${ }^{5}$ P. H. Bucksbaum, ${ }^{6}$ J. Hajdu, ${ }^{2,6}$ and H. Merdji ${ }^{1,6, *}$ \\ ${ }^{1}$ Commissariat à l'Energie Atomique, Service des Photons, Atomes et Molécules, \\ Bâtiment 522, Centre d'Etude de Saclay, 91191 Gif-sur-Yvette, France \\ ${ }^{2}$ Laboratory of Molecular Biophysics, Department of Cell and Molecular Biology, Uppsala University, \\ Husargatan 3 (Box 596), SE-751 24 Uppsala, Sweden \\ ${ }^{3}$ Laboratoire de Photonique et Nanostructures, CNRS-UPR20, Route de Nozay, F-91460 Marcoussis, France \\ ${ }^{4}$ Instituto de Plasmas e Fusão Nuclear, Instituto Superior Tecnico, Avenue Rovisco Pais, 1049-001 Lisboa, Portugal \\ ${ }^{5}$ Laboratoire d'Optique Appliquée, Ecole Nationale Supérieure de Technique Avancées, Ecole Polytechnique, CNRS UMR7639, \\ Chemin de la Hunière, 91761 Palaiseau Cedex, France \\ ${ }^{6}$ PULSE Institute, Stanford Linear Accelerator Center, Stanford University, 2575 Sand Hill Road, Menlo Park, California 94025, USA
}

(Received 30 January 2009; published 8 July 2009)

\begin{abstract}
Coherent x-ray diffractive imaging is a powerful method for studies on nonperiodic structures on the nanoscale. Access to femtosecond dynamics in major physical, chemical, and biological processes requires single-shot diffraction data. Up to now, this has been limited to intense coherent pulses from a free electron laser. Here we show that laser-driven ultrashort x-ray sources offer a comparatively inexpensive alternative. We present measurements of single-shot diffraction patterns from isolated nano-objects with a single 20 fs pulse from a table-top high-harmonic x-ray laser. Images were reconstructed with a resolution of $119 \mathrm{~nm}$ from the single shot and $62 \mathrm{~nm}$ from multiple shots.
\end{abstract}

PACS numbers: 87.59. $-\mathrm{e}, 41.50 .+\mathrm{h}, 42.65 .-\mathrm{k}$

Imaging individual objects, with a few nanometer resolution in space and a few femtosecond resolution in time, is of fundamental importance in many areas of science and represents today a fascinating challenge. In such context, advances in coherent diffraction using new coherent ultrashort $\mathrm{x}$-ray sources are actually demonstrating a high potential. Coherent diffraction imaging (CDI) overcomes many restrictions in imaging offering a means to produce images of noncrystalline objects at a resolution, in principle, limited by the wavelength only. In this "lensless" technique a spatially coherent $\mathrm{x}$-ray beam illuminates an isolated sample (surrounded by a zero-scattering area), and the far-field diffraction pattern of the object is recorded on an area detector. Since the detector records only the diffracted intensities the "real image" is retrieved from the amplitude and phase diffraction pattern using an iterative algorithm based on Fourier transforms [1]. This imaging technique can be scaled all the way down to atomic resolution. Since its demonstration, many researchers have taken large steps in this direction using soft and hard x-ray synchrotron radiation [2-6]. However, although atoms can be imaged in space, the conventional synchrotron sources do not allow accessing the fastest processes in matter, which is the new frontier of femtosecond to attosecond time resolution. It is only recently that this perspective has emerged thanks to the first demonstration of single-shot coherent diffraction imaging using a femtosecond soft $\mathrm{x}$-ray free electron laser (FEL) [7].
Following this work Barty and collaborators have then validated the time resolved approach in coherent diffractive imaging by capturing laser induced plasma expansion of a nanopatterned sample on picosecond time scales [8]. The key issue in such nonrepetitive experiments consists in recording diffraction patterns in a single shot with an ultrashort soft x-ray pulse. Indeed it has been proposed to use femtosecond or even shorter x-ray pulses to overcome the damage problems that would degrade $x$-ray images of individual nanoparticles: If a single ultrashort $\mathrm{x}$-ray pulse has enough $\mathrm{x}$ rays to form a good diffraction image of a nanoscale object, then the inertial confinement of the atoms during the ultrabrief exposure will prevent blurring, even if the $x$-ray flux is sufficient to destroy the sample [9]. However, this necessitates a very high coherent x-ray flux so far only available at large-scale limited-access FEL facilities [10]. This requirement limits the wide spread of ultrafast coherent diffraction imaging at the moment.

High-harmonic generation (HHG) would represent an excellent alternative since such sources are widely available and show the required properties [11-17] to perform ultrafast CDI: high spatial coherence, regular wave front, temporally coherent beam, and ultrashort pulse duration (femtosecond down to about 100 as). Moreover, HHG pulses are synchronized on subfemtosecond time scale with the driving infrared femtosecond laser, allowing a vast flexibility in time resolved experiments. Up to now HHG, like other laser based coherent soft x-ray sources, could not provide enough coherent flux to collect single- 
shot diffraction data. Their application was thus restricted to the investigation of nondestructive phenomena, where weak signals had to be accumulated over long time period (hours) $[18,19]$.

Here we report the first results on single-shot coherent diffraction imaging from a femtosecond table-top HHG source. Our approach is based on a significant improvement of the soft x-ray yield from high-harmonic generation in gases. The experiment was performed at the LUCA laser facility at CEA Saclay, France. The Ti:sapphire laser system delivers up to $50 \mathrm{~mJ}$ energy pulses at $800 \mathrm{~nm}$ with a pulse duration of $50 \mathrm{fs}$ at a repetition rate of $20 \mathrm{~Hz}$. A high intensity soft x-ray coherent beam is obtained using a loose focusing geometry, which allows coupling a very high amount of laser energy in the HHG process [20-22]. At a given laser intensity we increase the focal volume and scale up the number of "atomic harmonic converters" that will macroscopically radiate under improved phase matching conditions. Using a long gas cell and a long focal length lens $(7.5 \mathrm{~m})$, the emitting volume can be increased by orders of magnitude as compared to standard HHG setups. This approach allows reaching more than $10 \mu \mathrm{J}$ per shot in the whole harmonic frequency comb and up to $1 \mu \mathrm{J}\left(1.6 \times 10^{11}\right.$ photons $)$ for the 25 th $(\lambda=32 \mathrm{~nm})$ harmonic considered in this work. For the CDI experiment a laser energy of $\sim 35 \mathrm{~mJ}$ and a $5.5 \mathrm{~m}$ focal length lens were used. The soft x-ray beam parameters were optimized by adjusting the gas pressure, the cell length, and the laser beam aperture. The beam was focused into a $10 \mathrm{~cm}, 2 \mathrm{mbar}$ argon gas cell before the entrance of the cell. The maximum soft X-ray output was $0.6 \mu \mathrm{J}$. Thanks to a preferential on axis phase matching associated to the short quantum path [17], the beam has a low divergence (500 $\mu \mathrm{rad})$ and a high degree of spatial coherence, up to $92 \%$ as measured in single shots using a Young double slit arrangement.

The schematic arrangement of our table-top CDI beam line at CEA Saclay is shown in Fig. 1. After generation of the soft $\mathrm{x}$-ray beam, it propagates together with the main infrared (IR) laser which is then rejected using an IR antireflective mirror. The residual IR is eliminated using a $150 \mathrm{~nm}$ thick aluminum filter. A $22.5^{\circ}$ off axis parabola of $200 \mathrm{~mm}$ focal length focuses the soft $\mathrm{x}$ rays to a $20 \mu \mathrm{m}$ focal spot (full width at half maximum). The 25th harmonic is selected from the harmonic frequency comb using a $\mathrm{B}_{4} \mathrm{C} / \mathrm{Si}$ multilayer coating deposited on the parabola surface. The resulting beam has a spectral linewidth of $\lambda / \Delta \lambda \sim 150$ and a temporal duration of $\sim 20 \mathrm{fs}$. The total optic line transmission from the high-harmonic source to the sample is $1 \%$ corresponding to $10^{9}$ useful photons per shot at $32 \mathrm{~nm}$ in a $20 \mu \mathrm{m}$ focal spot. The intensity on the sample is estimated to be $10^{11} \mathrm{~W} / \mathrm{cm}^{2}$. The sample is placed at the focus of the parabolic mirror and diffracts the coherent soft $x$ rays. The samples were micrometersized "harmonic notes" with sub-100 nm details [see Figs. 1 and 4(a)]. To obtain a pure amplitude object an opaque $100 \mathrm{~nm}$ thick gold layer was deposited on a $70 \mathrm{~nm}$ thick SiC membrane before patterning using a sub-10 nm

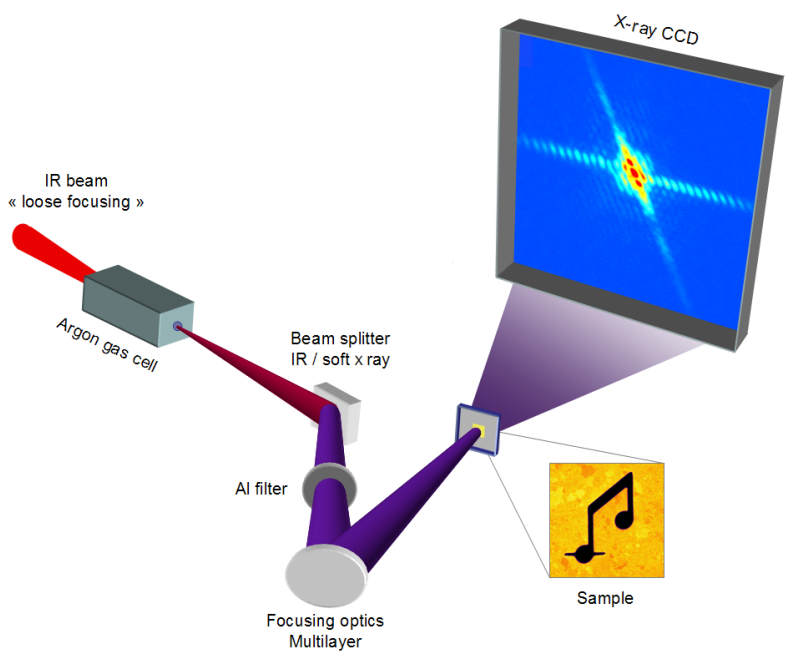

FIG. 1 (color online). Tabletop soft $x$-ray diffractive imaging beam line. The soft $\mathrm{x}$-ray laser harmonics are produced by the interaction of the loose focused infrared laser beam in an argon gas cell. The infrared beam is removed after the soft x-ray generation by a $\mathrm{SiO}_{2}$ beam splitter that reflects most of the soft x ray. An antireflective coating rejects most of the infrared laser light. The residual infrared light is totally suppressed with a $150 \mathrm{~nm}$ thin aluminum filter. The 25th harmonic order, at a wavelength of $32 \mathrm{~nm}$, is selected and focused onto the sample using a multilayer coated off axis parabola. The sample is positioned at the focus where it scatters the soft x-ray beam. The diffraction pattern is collected on a backilluminated soft x-ray CCD at $20 \mathrm{~mm}$ distance from the sample. With a $3 \mu \mathrm{m}$ sample size the diffraction pattern is measured in the Fraunhofer regime.

resolution focused ion beam. The diffraction pattern is collected on a backilluminated CCD camera in the Fraunhofer regime (at a distance $z=20 \mathrm{~mm}$ from the sample), where it is proportional to the Fourier transform of the sample's transmission function (the sample exit wave). The full chip contains $2048 \times 2048$ square pixels with a size of $p=13.5 \mu \mathrm{m}$.

To demonstrate the potential of our CDI beam line we have first considered multiple exposure diffraction patterns of the sample. A diffraction pattern of the harmonic notes is shown in Fig. 2(a). The data are recorded over 40 shots ( $2 \mathrm{~s}$ acquisition time), i.e., $10^{7}$ times less shots than what has been done previously using HHG. The image represents the coherent intensity pattern of the sample Fourier transform. In our experiment the use of a beam blocker was not necessary so that we have full access to low frequencies. This increases the amount of information for image reconstruction. The main diffracted features are distributed on two axes associated to the preferential directions in the sample structure. A 30 pixel period modulation can be seen on the horizontal direction. This is related to the interference of the diffracted waves from two similar features placed at a distance of $1.5 \mu \mathrm{m}$ in the sample design. The modulation is also observed at the largest diffracted angles. Practically it means that the diffracted beams overlap in time even at high angles. This is consistent with the spectral bandwidth and the pulse duration. 
Since only the intensity of the diffracted coherent wave is measured, the phase information is missing and must be recovered to reconstruct the object. Figure 2(b) shows the reconstructed image from the diffraction pattern given in Fig. 2(a). Image reconstruction was carried out using iterative transform phase retrieval techniques. Here, we used the HAWK code iterative procedure [23]. For both reconstructions, the experimental image was low pass filtered by applying a tight filter around the autocorrelation to remove most of the image noise. The support of the object was then determined using the SHRINKWRAP algorithm combined with hybrid input-output (HIO) algorithm. Using the relaxed averaged alternating reflectors (RAAR) algorithm and the support previously obtained, 80 reconstructions were run with random starting points.

In the Fraunhofer approximation, the maximal detectable spatial frequency in the sample's transmission function is calculated according to $\sigma_{\max }=(N p / 2) /(\lambda z)$, where $N$ represents the pixel number of the chip area considered. For the 40 shot exposure, an array of $N=$ $1024 \times 1024$ pixels is used for the reconstruction corresponding to a diffraction angle of $19^{\circ}$ at the midpoint of the
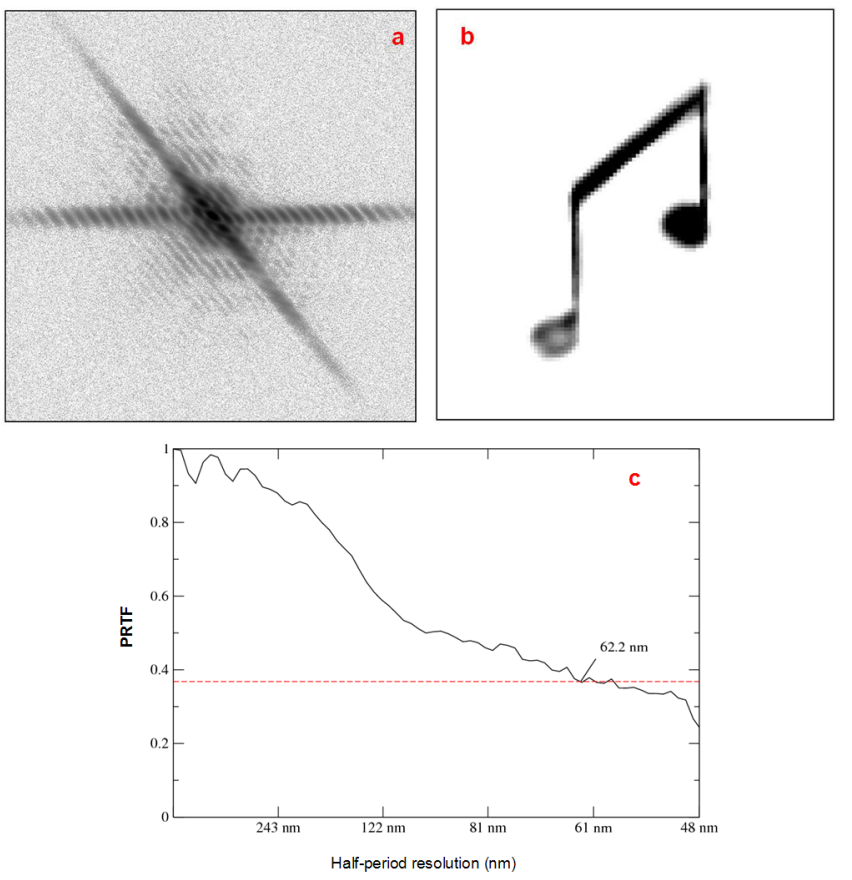

FIG. 2 (color online). Multishot coherent soft x-ray diffraction patterns and the reconstructed image. (a) Measured diffracted intensity (magnitude square of diffracted wave) from the sample, in logarithmic scale, obtained in 40 shots acquisition $(2 \mathrm{~s}$ exposure time). The maximal spatial frequency $\left(\sigma_{\max }\right)$ at the edge of the diffraction pattern is $10.8 \mu \mathrm{m}^{-1}$. (b) Reconstructed object amplitude obtained with a $46 \mathrm{~nm}$ pixel size. The reconstruction reveals the modification due to the damage that occurred to the object during the experimental campaign, in particular, at the round of the left harmonic note. (c) PRTF function of the image reconstructed in which the criteria value of $1 / e$ provides a halfperiod resolution length image of $62 \mathrm{~nm}$. edge of the array. The image resolution (based on Rayleigh criteria) corresponds to the half-period length of the highest frequency in the diffraction pattern. According to this definition the diffraction limited resolution $[r=$ $\left.1 /\left(2 \sigma_{\max }\right)\right]$ gives a pixel size of $46 \mathrm{~nm}$. The effective image resolution could be worse than $r$ depending on the accuracy with which the phases are retrieved. An effective halfperiod resolution is therefore estimated after reconstruction by computing the phase retrieval transfer function (PRTF) [4,7]. It is defined as the value where the PRTF drops to a value $1 / e$. According to this criteria, a $62 \mathrm{~nm}$ resolution length is obtained as shown in Fig. 2(c).

On the basis of this result, we have progressively lowered the exposure time from $2 \mathrm{~s}$ to $20 \mathrm{fs}$ corresponding to a single-shot acquisition. The measured diffraction pattern is shown in Fig. 3(a). In this case, the useful signal occupies a smaller region of the detector with less scattered photons on the detector compared to the multishot case. This limits the signal-to-noise ratio, and high frequencies are less evidenced. Signal-to-noise ratio limits the useful chip area for the reconstruction algorithm to an array of $N=$ $800 \times 800$ pixels corresponding to a diffraction angle $15^{\circ}$

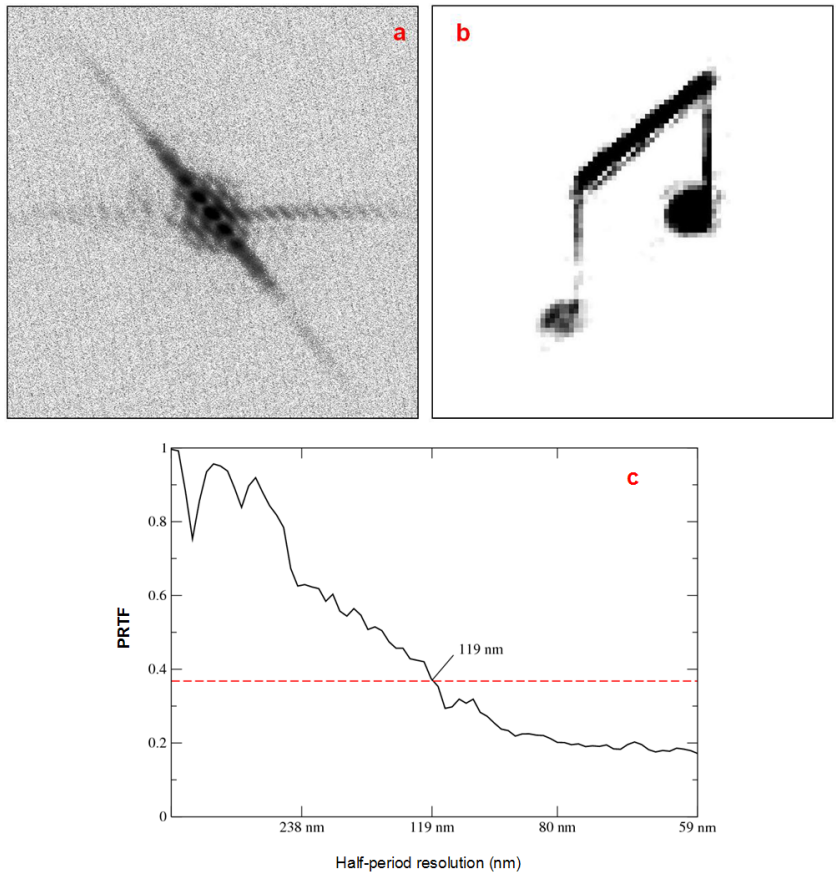

FIG. 3 (color online). Single-shot coherent soft x-ray diffraction patterns and the reconstructed image. (a) Measured diffracted intensity from the sample, in logarithmic scale, obtained in single-shot acquisition ( $20 \mathrm{fs}$ exposure time). The maximal spatial frequency at the edge of the diffraction pattern is $8.4 \mu \mathrm{m}^{-1}$. (b) Image of reconstructed object amplitude obtained with $59 \mathrm{~nm}$ pixel size. Despite less quality, the single-shot object reconstruction shows a qualitative agreement with the multishot reconstruction. In particular, the amplitude transmission is lower on the round of the left harmonic note. (c) PRTF function of the reconstructed image. The $1 / e$ criteria value provides a halfperiod resolution length image of $119 \mathrm{~nm}$. 


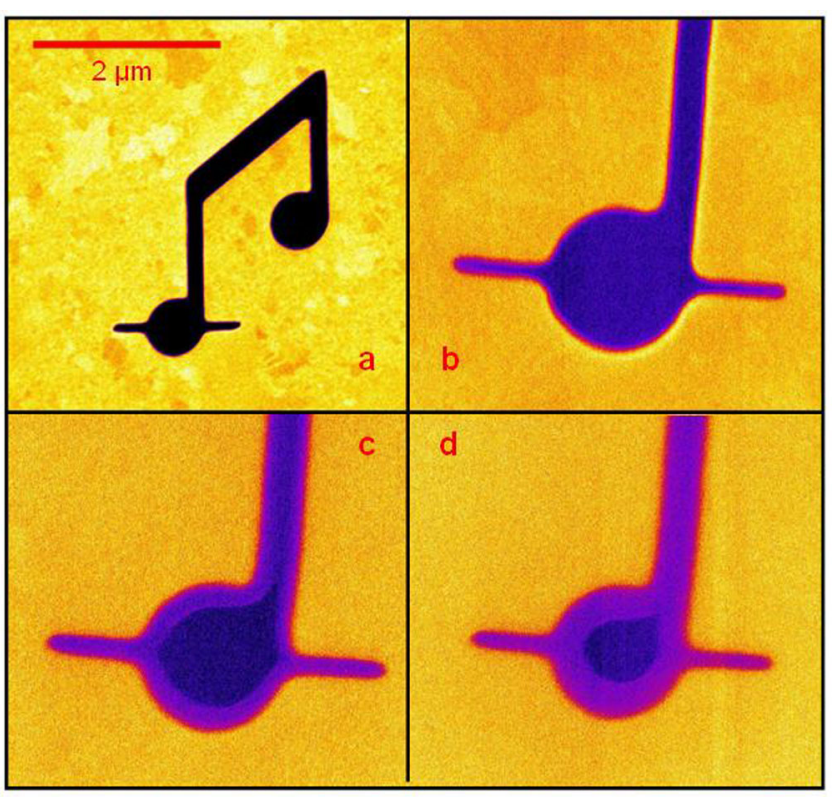

FIG. 4 (color online). "Harmonic notes" SEM images and details on the structure modification. Panel (a) shows the initial test object used at our table-top coherent imaging beam line. Panels (b)-(d) show a detail of the structure modification of the object at different steps during the experiment. These steps, respectively, correspond to a low (b), medium (c), and high (d) soft $\mathrm{x}$-ray dose. The dark (purple) area corresponds to a full transmission of the soft $\mathrm{x}$-ray whereas the bright (purple) area corresponds to a partial transmission. Panel (c) corresponds to the sample used to collect the diffraction patterns shown in Figs. 2(a) and 3(a).

at the midpoint of the edge of the array. However, the quality of the data is high enough to allow a reconstruction shown in Fig. 3(b). The features already evidenced in the multiexposure case are here similarly reconstructed. The PRTF function gives a resolution length of $119 \mathrm{~nm}$ [see Fig. 3(c)]. For both single and multishot images, there is a qualitative consistency with the scanning electron microscopy (SEM) image shown in Fig. 4(a). In particular, the two notes are well defined, as well as the upper bar (horizontally tilted thick line). However, the save line never appears, even if its thickness $(75 \mathrm{~nm})$ is larger than the multiexposure resolution. This is explained by considering the radiation damages progressively induced during the whole experimental campaign. While damages do not occur on single or few tens shots, the irradiation over thousands of shots (i.e., repetitive measurements) does affect the sample. In Figs. 4(b)-4(d) we show SEM images of the sample submitted to different radiation doses at different stages of the experiment. The evolution of the save line feature is pointed out. At the beginning of the experiment [Fig. 4(b)], the note is fully transmitting. When increasing the dose the transmitting area is progressively reduced as shown, respectively, in Figs. 4(c) and 4(d). The image shown in Fig. 4(c) corresponds to sample condition at the time when the diffraction pattern shown in Figs. 2(a) and 3(a) have been measured. The transmitting area is now much reduced, affecting both the circle diameter and the vertical line thickness. Moreover, the save line is completely blocked, in agreement with the reconstructed images in Figs. 2(b) and 3(b). These observations underline the importance of recording the full information before the sample is modified [9]. Single-shot femtosecond imaging allows data collection faster than relevant damages processes occur. This possibility, first demonstrated at the FLASH FEL, is now available on a table-top laser harmonic source.

In this Letter we have shown that HHG can deliver coherent intense $\mathrm{x}$ rays at the microjoule level. Our laboratory scale source offers an inexpensive approach, complementary to FEL facilities. We have demonstrated the capability of visualizing in single-shot nonperiodic nanoscale objects using femtosecond coherent diffractive imaging. This opens fascinating perspectives in imaging dynamical phenomena to be spread over a large scientific community. Investigation of ultrafast phase transitions in mesoscopic systems, ultrafast spin reversals of magnetic nanodomains, or large molecule rearrangements in biological environments are some examples. The natural synchronization of high-harmonic generation with the driving laser allows subfemtosecond time jitter that would significantly facilitate time resolved studies.

Shorter wavelengths already demonstrated in HHG should soon allow reaching sub-10 nm spatial resolution in coherent diffractive imaging. Exciting future prospects also come from intense harmonics generated from laserplasma surfaces $[24,25]$. This coherent source holds the promise of generating very intense subnanometer subfemtosecond coherent $\mathrm{x}$ rays suitable for ultrafast coherent diffractive imaging.

We acknowledge financial support from the European Union (TUIXS, NEST-012843), the EU-FP6 XTRA (MRTN-CT-2003-505138), and LASERLAB (RII3-CT2003-506350) programs. We also acknowledge support from the U.S. Department of Energy through the Stanford PULSE Center. Additional support comes from the Triangle de la Physique, NanoSciences Ile-de-France, DFG Cluster of Excellence at the Munich Centre for Advanced Photonics, from the Virtual Institute Program of the Helmholtz Society, and from the Swedish Research Council. We are grateful to Elsa Abreu, Thierry Auguste, Stefan Haessler, Jacek Krzywinski, and Pascal Salières for help and discussions.

*hamed.merdji@cea.fr

[1] J. W. Miao, P. Charalambous, J. Kirz, and D. Sayre, Nature (London) 400, 342 (1999).

[2] I. K. Robinson et al., Phys. Rev. Lett. 87, 195505 (2001).

[3] J. W. Miao et al., Proc. Natl. Acad. Sci. U.S.A. 100, 110 (2003). 
[4] D. Shapiro et al., Proc. Natl. Acad. Sci. U.S.A. 102, 15343 (2005).

[5] M. A. Pfeifer, G. J. Williams, I. A. Vartanyants, R. Harder, and I. K. Robinson, Nature (London) 442, 63 (2006).

[6] B. Abbey et al., Nature Phys. 4, 394 (2008).

[7] H. N. Chapman et al., Nature Phys. 2, 839 (2006).

[8] A. Barty et al., Nat. Photon. 2, 415 (2008).

[9] R. Neutze et al., Nature (London) 406, 752 (2000).

[10] W. Ackerman et al., Nat. Photon. 1, 336 (2007).

[11] P. Salières, A. L'Huillier, Ph. Antoine, and M. Lewenstein, Adv. At. Mol. Opt. Phys. 41, 83 (1999).

[12] T. Ditmire et al., Phys. Rev. Lett. 77, 4756 (1996).

[13] L. Le Deroff, P. Salières, B. Carré, D. Joyeux, and D. Phalippou, Phys. Rev. A 61, 043802 (2000).

[14] R. Bartels et al., Science 297, 376 (2002).

[15] P. B. Corkum and F. Krausz, Nature Phys. 3, 381 (2007).
[16] J. Gautier et al., Eur. Phys. J. D 48, 459 (2008).

[17] H. Merdji et al., Phys. Rev. A 74, 043804 (2006).

[18] R. L. Sandberg et al., Phys. Rev. Lett. 99, 098103 (2007).

[19] R. L. Sandberg et al., Proc. Natl. Acad. Sci. U.S.A. 105, 24 (2008).

[20] J. F. Hergott et al., Phys. Rev. A 66, 021801 (2002).

[21] E. Takahashi, Y. Nabekawa, and K. Midorikawa, Opt. Lett. 27, 1920 (2002).

[22] E. Takahashi, Y. Nabekawa, T. Otsuka, M. Obara, and K. Midorikawa, Phys. Rev. A 66, 021802 (2002).

[23] Details about the Hawk code iterative procedure can be found at http://xray.bmc.uu.se/ filipe/?q=hawk/ whatishawk.

[24] B. Dromey et al., Phys. Rev. Lett. 99, 085001 (2007).

[25] C. Thaury et al., Nature Phys. 4, 631 (2008). 